\title{
Layout Optimization of Campus Bike-Sharing Parking Spots
}

\author{
Tangyi Guo $\mathbb{D},{ }^{1,2}$ Jie Yang, ${ }^{1}$ Liu He $\mathbb{D}^{1,2}$ and Kun Tang ${ }^{1,2}$ \\ ${ }^{1}$ School of Automation, Nanjing University of Science \& Technology, Nanjing 210094, China \\ ${ }^{2}$ MIIT Key Lab of Traffic Information Fusion \& System Control, Nanjing 210094, China \\ Correspondence should be addressed to Liu He; heliu@njust.edu.cn
}

Received 31 July 2020; Accepted 13 October 2020; Published 2 November 2020

Academic Editor: Dongfang Ma

Copyright (c) 2020 Tangyi Guo et al. This is an open access article distributed under the Creative Commons Attribution License, which permits unrestricted use, distribution, and reproduction in any medium, provided the original work is properly cited.

\begin{abstract}
The rapid development of bike sharing has posed some challenges to the traffic management on campus. The bike sharing on campus has problems such as messy parking, and some buildings in the peak hours have no bikes to borrow. Thus, alternative parking spots are proposed based on the layout principle of parking spots for bicycles. An optimization model of the layout of campus bike-sharing parking spots with travel time and construction cost as the optimization goal is established, and the branch and bound algorithm is used to solve the model. Finally, the study analysis is carried out by optimizing the layout of the bike-sharing parking spot of Nanjing University of Science and Technology. The results show that, after optimizing the layout of parking spots, the average travel time of users is reduced by $6.0 \%$, and the total construction cost is reduced by $27.3 \%$. While being convenient for campus bike-sharing users, it also provides scientific decision-making support for the campus traffic management.
\end{abstract}

\section{Introduction}

Bike sharing not only helps to alleviate urban traffic pressure but also generates tremendous energy in constructing a green transportation trip system. It also has unique advantages in solving the "last mile" problem [1]. Bike sharing helps to alleviate urban traffic pressure in building a green energy traffic system and has a unique advantage in solving the "last kilometer" problem [2,3]. The dockless bike sharing has got rid of the limitation of fixed parking piles and has the characteristics of small traffic capacity, flexible operation, good accessibility, and less investment [4], which has gradually covered most of the first- and second-tier cities in China, as well as Singapore, Washington, and other overseas cities. However, due to the regular change process of bikes demand, the bike-sharing system does not guarantee selfbalancing, resulting in the phenomenon that a large number of shared bikes are idle in some areas and no bikes are available in some areas [5]. Therefore, it is imperative and necessary to set up a reasonable and convenient fixed storage spot for shared bikes.
The main problem of optimizing the number of parking spots and layout of shared bicycles can be solved by selecting the optimal facility location and related resource allocation based on different optimization objectives and constraints. Some scholars consider maximum coverage to optimize the shared bike system to maximize the demand covered by the shared bike parking spot or meet the limits of available budgets [6]. With the overall imbalance between supply and demand for shared bikes, Hu et al. [7] adopted three optimization models based on CMCLP to optimize the system configuration to achieve maximum service coverage [8-10]. Due to the close connection between the parking spots of bike sharing and the surrounding infrastructure, some scholars have considered the influence of the surrounding environment on the layout of shared bikes. In view of the optimization of the layout of shared bikes parking spots in scenic areas, Guo et al. [11] considered the distribution of subway stations and bus stations around the scenic area, by proposing an optimization model based on clustering and greedy algorithms, and solved the problem with an optimization coverage rate 
of $89.2 \%$. In establishing the layout optimization model of the shared bikes parking spot, we can also consider the multiobjective optimization model such as travel cost and construction cost [12]. Romero et al. [13] proposed a twolayer mathematical programming model to optimize the location of the base station while minimizing total costs and maximizing the number of system users. Luis Ines Frade et al. [14] proposed the maximum coverage model from the perspective of user demand, with the goal of maximizing demand and the optimized model with the available budget and system benefits as the constraints, so as to get the optimal location and configuration of the parking spot. In addition, George and Xia [15] introduced a queuing theory in the study of the size of the shared bike rental spot. García-Palomares et al. [16] proposed a GIS-based approach to calculate the spatial distribution of potential travel demand, using the location allocation model to determine the location of shared bike parking spots, the capacity of the parking spots, and the demand characteristics of defining the parking spots. At present, the optimization of the bike-sharing layout mainly focuses on scenic spots and cities, which involves the maximum coverage maximization and cost minimization. The model solution involves clustering, particle swarm algorithm [17], genetic algorithm, and ant colony algorithm [18], while there are few studies on the layout optimization of bikesharing parking spots on campus.

Teachers and students are the main targets of bikesharing service within the campus. The phenomenon of stopping and parking anywhere is widespread within the campus which can easily lead to the problem of 'difficulty to find a bike' thereby reducing the convenience of the user's travel [19]. According to the characteristics of the parking spot of bike sharing on campus, this paper defines the rules for setting up the parking spots of shared bicycles on campus and establishes the distribution model of the parking spots of bike-sharing on campus with travel time and construction cost as the optimization goal. Finally, the layout plan of the bike-sharing parking spot on campus is designed with specific cases to verify the rationality and feasibility of the model.

\section{Problem Description}

Considering that the traffic operation status quo is of different areas and the geographical conditions and structure of residents' travel are also different, it goes without saying that users will have different needs for bike sharing. To begin with, we divide the different functional areas of the campus which are mainly the teaching areas, office areas, living areas, and activity areas, to make certain of the demand for each service area; then, for different service areas, taking into account the reasonable number and location of parking spots, the premise of meeting the bike-sharing needs of each parking spot, the total travel time, and the total construction cost are minimized, and the layout optimization model is perfectly built.

2.1. Parking Lots Layout Planning. According to different service properties of different functional buildings, the campus is divided into several areas that are conducive to the layout of bike-sharing parking spots, and in each divided area, alternative parking spots are set according to different building radiation ranges.

The selection of alternative parking spots for bike sharing is in accordance with the following principles:

(1) Maximum service radius of parking spots: researches have shown that most bike sharing use areas within 300 meters from the station [18], and the maximum service radius of bike-sharing parking spots is fixed to $300 \mathrm{~m}$, as shown in Figure 1

(2) Taking into consideration the nature of building services: the needs of people in different functional buildings for bike sharing are unpredictable, and the impact of user needs on the layout of parking spots should be fully considered

2.2. Layout Optimization Model. The aim of this model is to minimize the total travel time of users and spot construction costs on the premise of meeting the bike-sharing needs of each parking spot. The total travel time of users includes the total time of walking to the station, riding time, and time spent walking to the destination after returning the bike. The spot construction cost includes fixed cost of parking spot and cost of bikes. The model parameter symbols description is shown in Table 1.

The model objective functions are as follows:

$$
\begin{aligned}
& \min T=\sum_{i \in I} \sum_{i^{\prime} \in I}\left(\frac{\sum_{j \in J} b s_{i, j, i^{\prime}} \cdot d_{i, j}}{u_{1}}+\frac{\sum_{j \in J} \sum_{j^{\prime} \in J} b r_{i, j, j^{\prime}, i^{\prime}} \cdot d_{j, j^{\prime}}}{u_{2}}+\frac{\sum_{j^{\prime} \in J} r s_{i, j^{\prime}, i^{\prime}} \cdot d_{i^{\prime}, j^{\prime}}}{u_{1}}\right), \\
& \min P=\sum_{j \in J} y_{j} \cdot c_{0}+\sum_{j \in J} y_{j} \cdot b n_{j} \cdot c_{1}, \\
& \min \psi=k \cdot T+(1-k) \cdot P .
\end{aligned}
$$




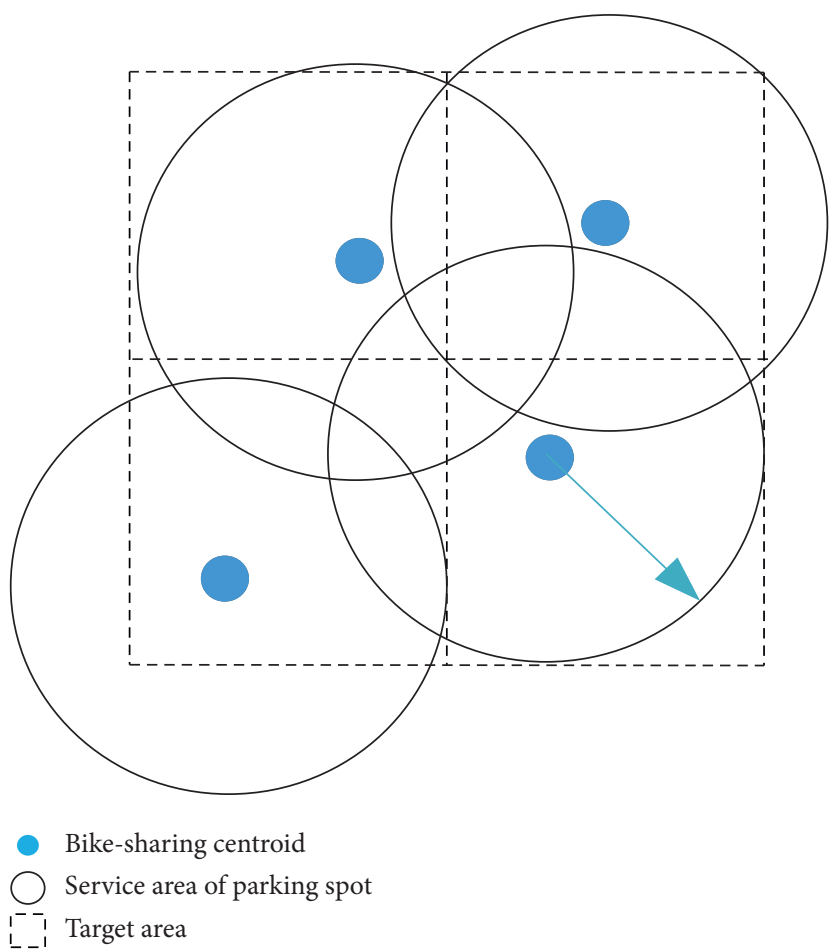

FIGURE 1: Diagram of the service scope of the bike-sharing parking spots.

TABle 1: Model parameter symbols description.

\begin{tabular}{|c|c|}
\hline ara & \\
\hline$I$ & Set of target areas, where, $i, i^{\prime} \in I$ \\
\hline$J$ & Set of alternative bike-sharing parking spots, where, $j, j^{\prime} \in J$ \\
\hline$k$ & Weight of the objective function \\
\hline$y_{j}$ & A binary variable to determine whether the parking spot is optimized, and the value of the optin \\
\hline$b s_{i, j, i^{\prime}}$ & $\begin{array}{l}\text { The number of the users depart from the target area } i \text { to the target area } i^{\prime} \text {, select borrow bicycles at the parking spot } j \text {, and the } \\
\text { travel route is } i \longrightarrow j \longrightarrow i^{\prime} \text {, where, } i, i^{\prime} \in I, j \in J\end{array}$ \\
\hline$b n_{j}$ & The number of bikes per spot \\
\hline$r s_{i, j^{\prime}, i^{\prime}}$ & $\begin{array}{l}\text { The number of the users depart from the target area } i \text { to the target area } i^{\prime} \text {, select return bikes at the parking spot } j^{\prime} \text {, and the } \\
\text { travel route is } \longrightarrow j^{\prime} \longrightarrow i^{\prime} \text {, where, } i, i^{\prime} \in I, j^{\prime} \in J\end{array}$ \\
\hline$b r_{i, j, j^{\prime}, i^{\prime}}$ & $\begin{array}{l}\text { The number of the users depart from the target area } i \text { to the target area } i^{\prime} \text {, select borrow bicycles at the parking spot } j \text {, and select } \\
\text { return bicycles at the parking spot } j^{\prime} \text {. The travel route is } i \longrightarrow j \longrightarrow j^{\prime} \longrightarrow i^{\prime} \text {, where, } i, i^{\prime} \in I, j \in J\end{array}$ \\
\hline$d_{i, j}$ & Distance from the target area $i$ to the parking spot $j$, where, $i \in I, j \in J$ \\
\hline$d_{j, j^{\prime}}$ & Distance from the parking spot $j$ to the parking spot $j^{\prime}$, where, $j, j^{\prime} \in J$ \\
\hline$d_{i^{\prime}, j^{\prime}}$ & $i^{\prime}$ to the parking spot $j^{\prime}$, where, $i^{\prime} \in I, j^{\prime} \in J$ \\
\hline$S_{\text {min }}, S_{\text {ma }}$ & The lower limit and upper limit of the number of alternative construction parking spots \\
\hline C & f 1 lo \\
\hline$\alpha_{i, j}$ & $\begin{array}{l}\text { A binary variable determines whether the target area } i \text { is within the service range of the optimized parking spot } j \text {; when the } \\
\text { target area is within the service range of the parking spot, the value is } 1 \text {, where, } i \in I, j \in J\end{array}$ \\
\hline$u_{1}, u_{2}$ & \\
\hline$M$ & 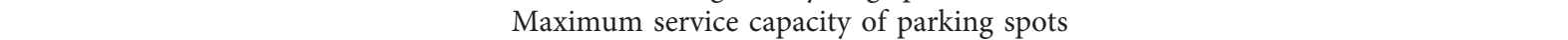 \\
\hline$D_{i, i^{\prime}}$ & The number of vehicles from target area ito target area $i^{\prime}$ \\
\hline
\end{tabular}

Equation (1) is the objective function, that is, the smallest total travel time of the user, including the walking time, borrowing and returning the bike, and riding time. Equation (2) is the objective function of construction cost. Equation (3) is the synthetic objective function. Restrictions:

$$
\begin{aligned}
S_{\min } & \leq \sum_{j \in J} y_{j} \leq S_{\max }, \\
\alpha_{i, j} & =\left\{\begin{array}{l}
1, d_{i, j} \leq C \\
0, d_{i, j}>C
\end{array}, \quad \forall i \in I, \forall j \in J,\right.
\end{aligned}
$$


$\sum_{j \in J} \alpha_{i, j} \cdot y_{j} \geq 1, \quad \forall i \in I$,

$\sum_{i \in J} \alpha_{i, j} \geq 1, \quad \forall j \in J$

$b s_{i, j, i^{\prime}} \leq M \alpha_{i, j} y_{j}, \forall i, i^{\prime} \in I$, and $i \neq i^{\prime}, \quad \forall j \in J$,

$r s_{i, j^{\prime}, i^{\prime}} \leq M \alpha_{i^{\prime}, j^{\prime}} y_{j}, \forall i, i^{\prime} \in I$, and $i \neq i^{\prime}, \quad \forall j^{\prime} \in J$,

$b s_{i, j, i^{\prime}}=\sum_{j^{\prime} \in J} b r_{i, j, j^{\prime}, i^{\prime}}, \forall i, i^{\prime} \in I$, and $i \neq i^{\prime}, \quad \forall j \in J$, and $j \neq j^{\prime}$,

$r s_{i, j^{\prime}, i^{\prime}}=\sum_{j \in J} b r_{i, j, j^{\prime}, i^{\prime}}, \forall i, i^{\prime} \in I$, and $i \neq i^{\prime}, \quad \forall j^{\prime} \in J$, and $j \neq j^{\prime}$,

$\sum_{j \in J} b s_{i, j, i^{\prime}} \geq D_{i, i^{\prime}}, \forall i, i^{\prime} \in I$

$y_{i}=\{0,1\}, \quad \forall j \in J$

$\sum_{j \in J} \alpha_{i, j}, b n_{j}, y_{j} \geq D_{i}$

Equation (4) is to optimize the construction of the number of bike-sharing parking spots, to avoid the situation where the number of parking spots is too small or too much, resulting in inefficient use of shared bike systems or resource redundancy; equation (5) is the target area $\mathrm{i}$ within the service area of the optimized parking spot j' s scope of services of binary variable, that is, to ensure that the user can find the parking spot within the maximum tolerable walking distance to complete the borrowing and returning of the bike within the given target area; equation (6) provides a bike borrowing and returning service for at least one bike-sharing parking spot in any target area; equation (7) serves at least one target area for a shared bike parking spot; equations (8) and (9) restrict users to borrow and return bikes at only optimized parking spots; equation (10) constrains the number of borrowed bikes at any one parking spot to be equal to the sum of the number of returned bikes from any parking spot to each parking spot; equation (11) constrains the number of bikes returned at any one parking spot to be equal to the sum of the number of vehicles returned from each parking spot to any one of the parking spots; equation (12) constrains the number of vehicles demanded from each target area to travel to other areas; equation (13) is the binary variable to optimize the parking spot; and equation (14) ensures that the number of vehicles in the target area meets the demand in the target area.

\section{Model Solution}

Owing to the fact that branch and brand is a basic method for solving integer planning (or mixed integer planning) in operations, it is very efficient to seek the optimal solution of integers. In this paper, the model is solved by the branch and bound method, and the solving steps are as follows:

Step 1: first, the integer constraints of the original problem are not considered, to solve the corresponding relaxation problem, and a graph or simplex method is used to obtain the optimal solution $Z$.

Step 2: if the optimal solution sought is just the integer solution, the integer solution is the optimal solution of the original integer planning problem.

Step 3: branch: based on our understanding of the importance of variables, we select a value that does not meet the integer constraint $X_{j}$ in the optimal solution, its value is $b_{j}$, where $\left[b_{j}\right]$ represents the maximum number which is less than $b_{j}$. Two constraints were constructed, $X \leq b_{j}$ and $X \geq\left[b_{j}\right]+1$, joining the original LP problem separately which formed two subproblems because $\left[b_{j}\right]$ and $\left[b_{j}\right]+1$ have no integers between them, so the integer solution within these two subsets must be consistent with the original feasible solution of the whole number solution.

Step 4: bounding: first, we determine whether there is an integer solution to each subproblem. If there is one, we find out the integer solution corresponding to the maximum value of the target function, fixed as $Z^{*}$, and then, the integer solution of the problem is the target function $Z \geq Z^{*}$, which is the bounding. Also, in the branching process, once there is a subproblem, then $Z^{*}=Z$.

Step 5: if there is a subproblem greater than $Z^{*}$, that needs to be branched out. If there is no integer solution in Step 4, it is also necessary to continue to branch to find the integer solution and to branch the subproblem corresponding to the maximum value of the target function.

Step 6: if the target value of all subproblems is less than or equal to $Z^{*}$, there is no need to continue branching, and the corresponding integer solution for $Z^{*}$ is the optimal solution.

The solution process of branch and bound algorithm is shown in Figure 2. 


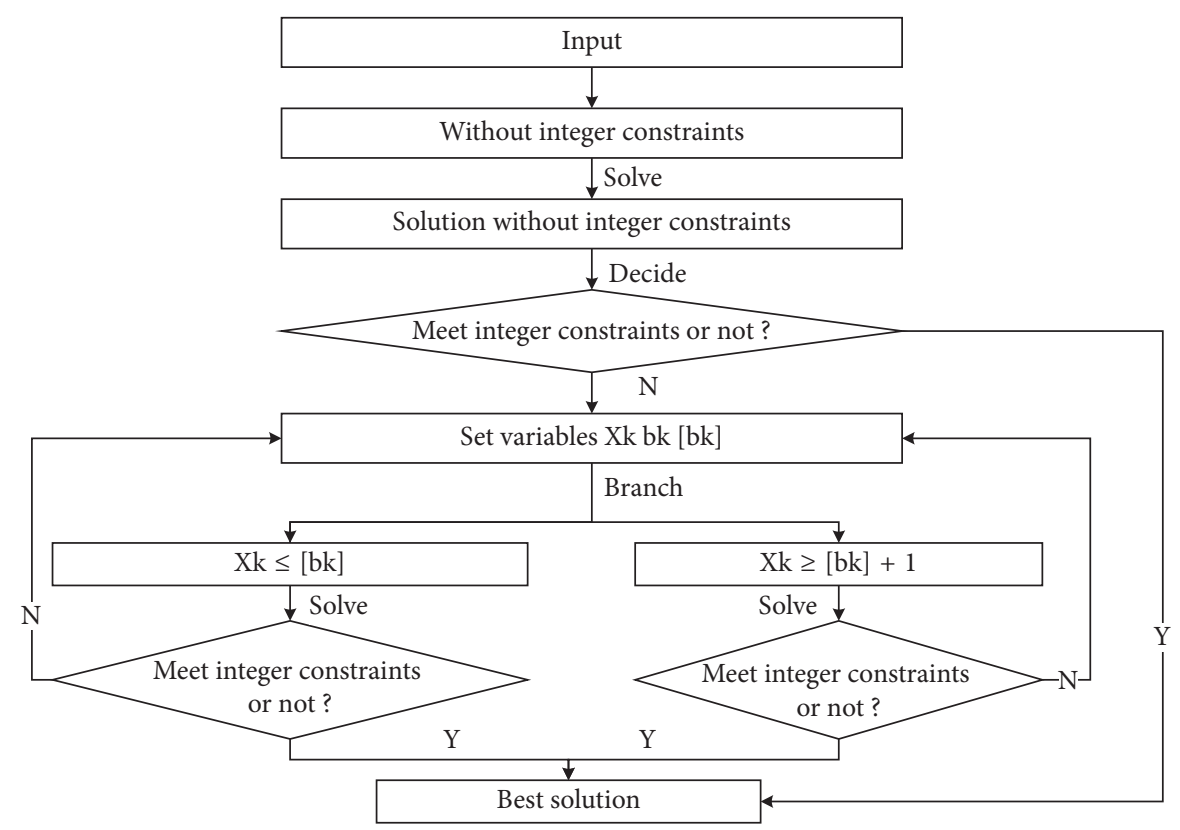

FIGURE 2: Solution process of branch and bound algorithm.

\section{Case Study}

In order to verify the accuracy of the layout optimization model of shared bike parking spots and the accuracy of the scheduling optimization model and the effectiveness of solving the genetic algorithm of the abovementioned model, this paper analyzes the genetic algorithm of Nanjing University of Technology as the research object. The school covers an area of 3118 acres, there are more than 30,000 students and more than 3,200 staff, and the travel demand is comparatively greater.

First of all, a statistical assessment was carried out on the number of people $N_{i}$ working in the functional buildings and regions who travel during peak hours. The distribution of alternative parking spots is shown in Figure 3. Different needs for bike sharing for users in different regions are used to calculate the demand for bikes. The percentage of bikes used for different regions is based on the analysis of survey data, as is shown in Table 2.

$$
\operatorname{Demand}_{i}=N_{i} * b_{i} \text {, }
$$

where $b_{i}$ is the percentage of bikes used for each functional region.

Considering the fact that the teaching area, office area, living area 1 and living area 3, and other space range are large, in order to ensure the reasonable distribution of parking spots and the use of grid layout, taking into account that the service radius of the parking spot is $300 \mathrm{~m}$, the set style size is $300 \mathrm{~m} \times 300 \mathrm{~m}$, to set the target regional center spot coordinates, as shown in Figure 4 . Then, the target regional center spots and coordinate values of the alternate parking spots and the shared bike demand between the target areas are entered separately as the parking spot layout optimization model input data.
The parking spot layout optimization model parameter values are as shown in Table 3.

In a global optimal angle, the travel time of all users is the target function, and the layout scheme with the smallest total travel time and the total construction cost is solved. Based on the branch boundary method, this paper uses Matlab to write a program to solve the model, and the optimization results are shown in Figure 5. After optimization, the number of shared bike parking spots has been reduced from 51 to 35 , which effectively reduces the operating and maintenance costs of shared bike parking spots.

Because of the convenience of the pile-free shared bikes, when considering the site construction cost, there is no need to consider the cost of building a parking pile, so the site construction cost only considers the fixed cost and the bike operation and maintenance cost.

$$
P=\sum_{j \in J} y_{j} \cdot c_{0}+\sum_{j \in J} y_{j} \cdot b n_{j} \cdot c_{1},
$$

where $P$ is the total construction cost, $y_{j}$ is the binary variable of the alternative site to optimize the site, $b n_{j}$ represents the number of bikes per spot, $c_{0}, c_{1}$, respectively, are the cost of construction of a single site and the cost of operation and maintenance of a single bike, $c_{0}$ equals 200 Yuan/unit, and $c_{1}$ equals 500 Yuan/bike.

Compared with the optimization model that only considers the shortest travel time, the model proposed in this paper reduces the average travel time of users by $6.0 \%$ and reduces the total construction cost by $27.3 \%$. The comparative analysis of optimization results is presented in Table 4. The number of optimized parking spots and corresponding shared bikes is shown in Table 5. The 


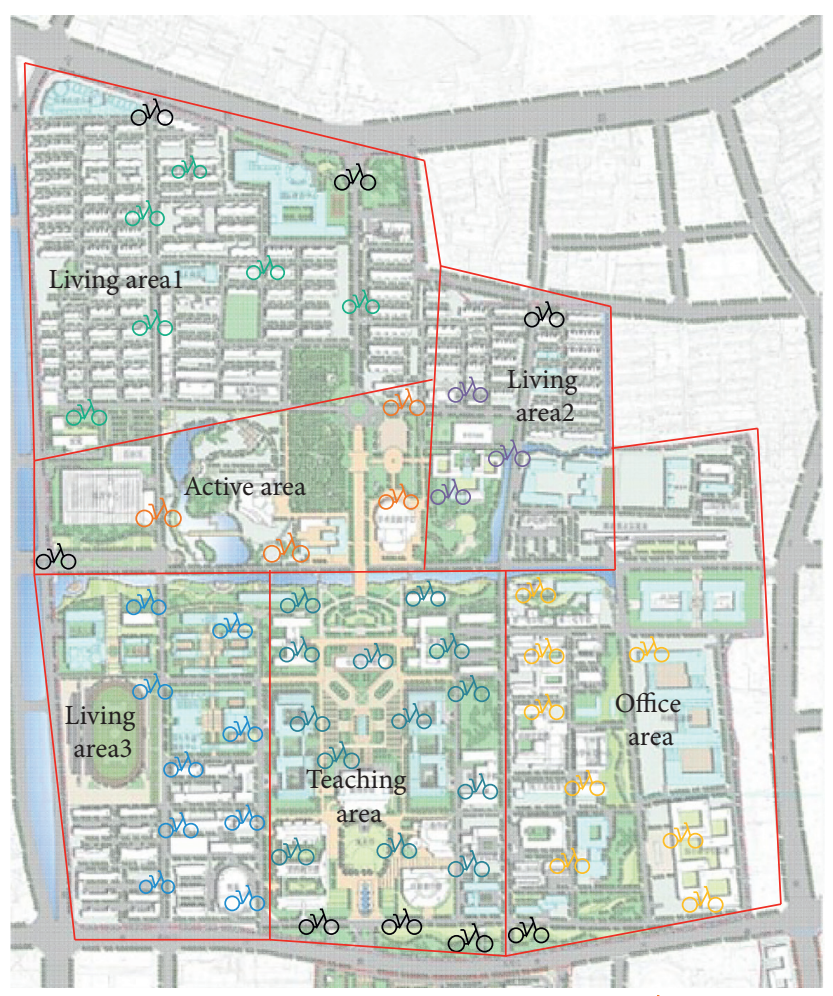

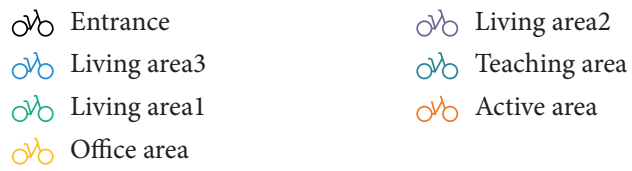

FIGURE 3: Distribution of alternative parking spots at Nanjing University of Science and Technology.

TABle 2: Campus zoning planning.

\begin{tabular}{|c|c|c|c|c|c|c|}
\hline No. & Zone & Spot & Number of people travelling & Bicycle ratio & Demand & Number of spots \\
\hline 1 & \multirow{8}{*}{ Entrance } & Gate 1 & 170 & 0.22 & 37 & \multirow{8}{*}{8} \\
\hline 2 & & Gate 2 & 34 & 0.22 & 7 & \\
\hline 3 & & Gate 3 & 272 & 0.22 & 60 & \\
\hline 4 & & Gate 7 & 170 & 0.22 & 37 & \\
\hline 5 & & South gate & 340 & 0.22 & 75 & \\
\hline 6 & & Gate 5 & 170 & 0.22 & 37 & \\
\hline 7 & & Underground tunnel 1 & 816 & 0.22 & 180 & \\
\hline 8 & & Underground tunnel 2 & 850 & 0.22 & 187 & \\
\hline 9 & \multirow{6}{*}{ Living area 1} & Xiyuan District 1 & 340 & 0.15 & 51 & \multirow{6}{*}{6} \\
\hline 10 & & Huayuan District & 680 & 0.15 & 102 & \\
\hline 11 & & Zhongshan District & 680 & 0.15 & 102 & \\
\hline 12 & & Campus hospital & 272 & 0.15 & 41 & \\
\hline 13 & & Zhuyuan District & 170 & 0.15 & 26 & \\
\hline 14 & & Xiyuan District 2 & 136 & 0.15 & 20 & \\
\hline 15 & \multirow{3}{*}{ Living area 2} & Keyuan District & 510 & 0.13 & 66 & \multirow{3}{*}{3} \\
\hline 16 & & Zilu hotel & 56 & 0.13 & 2 & \\
\hline 17 & & Postgraduate canteen & 272 & 0.13 & 35 & \\
\hline 18 & \multirow{4}{*}{ Active area } & Academic Ex. Center & 204 & 0.11 & 22 & \multirow{4}{*}{4} \\
\hline 19 & & Art and Culture Museum & 68 & 0.11 & 7 & \\
\hline 20 & & Sports center & 272 & 0.11 & 30 & \\
\hline 21 & & School of Foreign Language & 306 & 0.20 & 61 & \\
\hline
\end{tabular}


TABle 2: Continued.

\begin{tabular}{|c|c|c|c|c|c|c|}
\hline No. & Zone & Spot & Number of people travelling & Bicycle ratio & Demand & Number of spots \\
\hline 22 & \multirow{8}{*}{ Office area } & Sch. of Computer Sci. & 782 & 0.20 & 156 & \multirow{8}{*}{8} \\
\hline 23 & & Sch. of Electrical and Optical Sci. & 816 & 0.20 & 163 & \\
\hline 24 & & Sch. of Chemical Eng. & 408 & 0.20 & 82 & \\
\hline 25 & & Transient physical state & 17 & 0.20 & 3 & \\
\hline 26 & & Key labs & 17 & 0.20 & 3 & \\
\hline 27 & & Civil Explosion building & 17 & 0.20 & 3 & \\
\hline 28 & & Intelligent building & 340 & 0.20 & 4 & \\
\hline 29 & & Basic laboratory building 1 & 374 & 0.20 & 4 & \\
\hline 30 & \multirow{13}{*}{ Teaching area } & Basic laboratory building 2 & 680 & 0.21 & 143 & \multirow{13}{*}{13} \\
\hline 31 & & Teaching building 1 & 782 & 0.21 & 164 & \\
\hline 32 & & Teaching building 2 & 816 & 0.21 & 171 & \\
\hline 33 & & Teaching building 3 & 680 & 0.21 & 143 & \\
\hline 34 & & Teaching building 4 & 340 & 0.21 & 71 & \\
\hline 35 & & Yifu building & 340 & 0.21 & 71 & \\
\hline 36 & & Library & 170 & 0.21 & 36 & \\
\hline 37 & & Sport Gallery & 204 & 0.21 & 43 & \\
\hline 38 & & Qian Xuesen College & 340 & 0.21 & 71 & \\
\hline 39 & & Zhiyuan building & 306 & 0.21 & 64 & \\
\hline 40 & & Materials research center & 170 & 0.21 & 36 & \\
\hline 41 & & Printing plant & 170 & 0.21 & 36 & \\
\hline 42 & & Experiment center & 34 & 0.21 & 7 & \\
\hline 43 & \multirow{9}{*}{ Living area3 } & Zhizhen building & 170 & 0.12 & 20 & \multirow{9}{*}{9} \\
\hline 44 & & Youth League building & 340 & 0.12 & 41 & \\
\hline 45 & & 2 and 3 canteen & 408 & 0.12 & 49 & \\
\hline 46 & & Ming Yuan & 340 & 0.12 & 41 & \\
\hline 47 & & Campus supermarket & 306 & 0.12 & 37 & \\
\hline 48 & & Gymnasium & 306 & 0.12 & 37 & \\
\hline 49 & & 204 Student Dormitory & 340 & 0.12 & 41 & \\
\hline 50 & & Int. Students Dormitory & 612 & 0.12 & 73 & \\
\hline 51 & & Huizhi Pavilion & 680 & 0.12 & 82 & \\
\hline
\end{tabular}

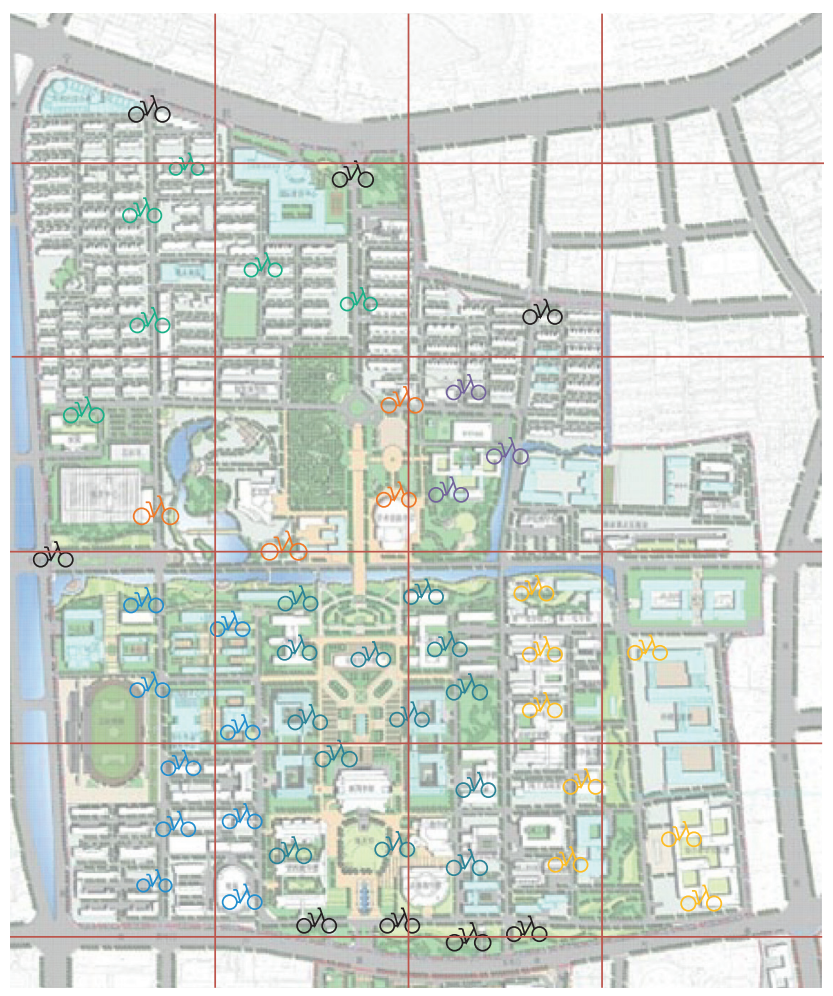

Figure 4: Grid zoning. 
TABle 3: Parameter values of the optimized model of the parking spot layout.

\begin{tabular}{lcccccc}
\hline Parameter & $C$ & $u_{1}$ & $u_{2}$ & $M$ & $S_{\min }$ & \\
\hline Value & 300 & 1.4 & 5 & 1000 & 25 & 40 \\
\hline
\end{tabular}

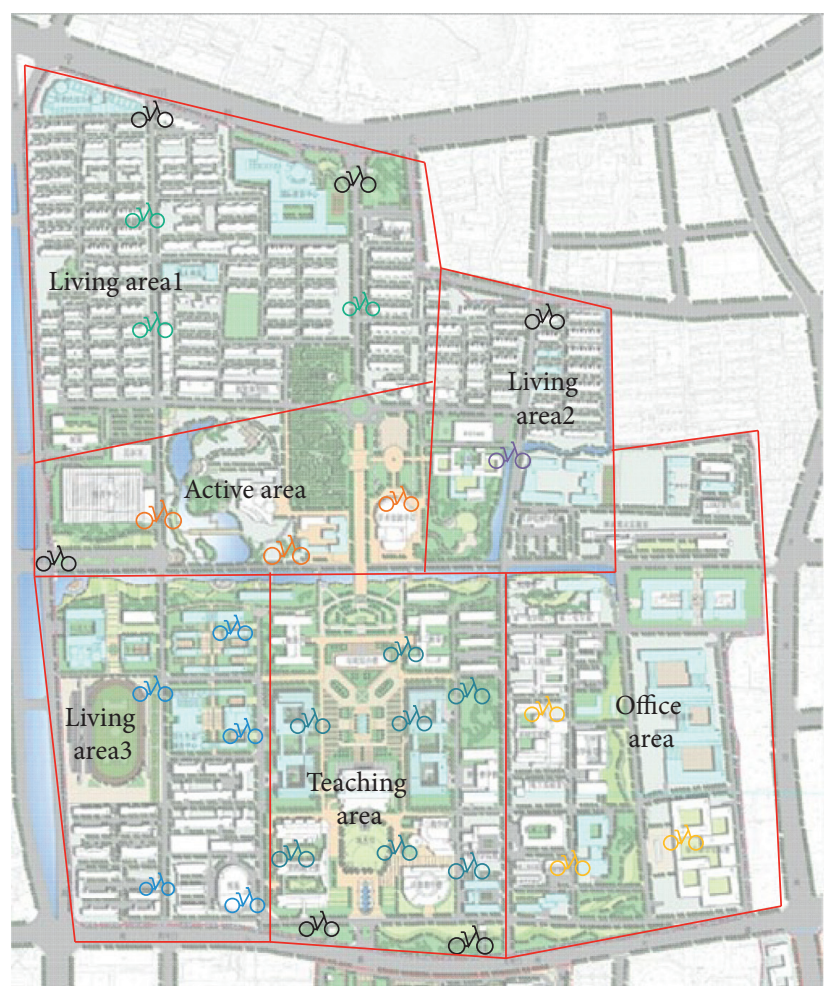

oto Entrance

ㄱo Living area3

oto Living area1

oto Office area oro Living area2

oro Teaching area

oblo Active area

FIgURe 5: Planning and layout of optimized parking spots.

TABLE 4: Comparative analysis of optimization results.

\begin{tabular}{lcc}
\hline Parameter & Research model & Contrast model \\
\hline Total travel time/s & 418489.6 & 394822.6 \\
Total construction cost/Yuan & 671600 & 923500 \\
Objective function value & 545044.8 & 659161.3 \\
The number of shared bike parking spots & 28 & 35 \\
\hline
\end{tabular}

TABLE 5: Number of optimized parking spots and corresponding shared bikes.

\begin{tabular}{|c|c|c|c|c|c|c|c|c|c|c|}
\hline Parking spot & 1 & 2 & 3 & 4 & 5 & 6 & 7 & 8 & 9 & 10 \\
\hline Number of shared bikes & 37 & 7 & 60 & 37 & 75 & 51 & 102 & 26 & 6 & 27 \\
\hline Parking spot & 11 & 12 & 13 & 14 & 15 & 16 & 17 & 18 & 19 & 20 \\
\hline Number of shared bikes & 27 & 61 & 163 & 3 & 3 & 37 & 68 & 78 & 82 & 68 \\
\hline Parking spot & 21 & 22 & 23 & 24 & 25 & 26 & 27 & 28 & & \\
\hline Number of shared bikes & 34 & 34 & 17 & 17 & 40 & 44 & 40 & 88 & & \\
\hline
\end{tabular}


TABle 6: The optimization result when $k$ takes different values.

\begin{tabular}{lccc}
\hline Parameter & Total travel time/s & Total construction cost/Yuan & The number of shared bike parking spots \\
\hline$k=0.1$ & 483624 & 644300 & 24 \\
$k=0.2$ & 445960 & 651900 & 27 \\
$k=0.3$ & 445855 & 651900 & 27 \\
$k=0.4$ & 436092 & 656700 & 26 \\
$k=0.5$ & 418490 & 671600 & 28 \\
$k=0.6$ & 409266 & 682000 & 30 \\
$k=0.7$ & 409266 & 682000 & 30 \\
$k=0.8$ & 401180 & 703900 & 32 \\
$k=0.9$ & 401180 & 703900 & 32 \\
\hline
\end{tabular}

planning and layout of optimized parking spots are shown in Figure 5.

This paper also analyzes the influence of the weight $k$ value in the model on the layout optimization results, as shown in Table 6 . The results show that the value of $k$ determines the impact of the total travel time and construction cost on the objective function, so the corresponding optimization results are quite different. When $k=0.1$, the optimized parking point is 24 ; when $k=0.9$, the optimized parking point is 32 .

\section{Conclusions}

(1) Considering the influence of the functionality of campus buildings on the demand of bike sharing, the layout rules of the bike-sharing parking spot are proposed.

(2) In view of the optimization of the campus bikesharing parking spot layout, a model of campus bikesharing parking spot planning is established with the total travel time of the user and the total construction cost as the optimization goal.

(3) The rationality of the model is verified by using Nanjing University of Science and Technology as the research case study. The user's travel time is reduced by $6.0 \%$, the total construction cost is reduced by $27.3 \%$, and it has a certain reference value for the operation management of bikesharing on campus.

The results also show that considering different factors will have a greater impact on the optimization results. There are many factors that affect the layout of campus bike-sharing parking spots. In the subsequent research work, the planning of shared bicycle parking spots under multiobjective and multiconstraint conditions can be considered to further improve the model.

\section{Data Availability}

On request, the data supporting the results of this study can be provided by the author of the article.

\section{Conflicts of Interest}

The authors affirm that there are no conflicts of interest.

\section{Acknowledgments}

This research was supported by "the Fundamental Research Funds for the Central Universities" (Nos. 30920010010 and 30919011290).

\section{References}

[1] H. Zhang, X. Song, T. Xia et al., "MaaS in bike-sharing: smart phone GPS data based layout optimization and emission reduction potential analysis," Energy Procedia, vol. 152, pp. 649-654, 2018.

[2] Z. Zheng, C. Lei, and Y. Ouyang, "Optimal investment and management of shared bikes in a competitive market," Transportation Research Part B, vol. 135, 2020.

[3] S. A. Shaheen, A. P. Cohen, and E. W. Martin, "Public bikesharing in north America, early operator understanding and emerging trends," Transportation Research Record, vol. 2387, no. 1, 2013.

[4] Y. Liu, W. Y. Szeto, and S. C. Ho, "A static free-floating bike repositioning problem with multiple heterogeneous vehicles, multiple depots, and multiple visits," Transportation Research Part C, vol. 92, 2018.

[5] S. A. Shaheen, S. Guzman, and H. Zhang, "Bikesharing in europe, the americas, and asia: past, present, and future," Transportation Research Record Journal of the Transportation Research Board, vol. 2143, pp. 159-167, 2019.

[6] S. Davari, M. H. Fazel Zarandi, and I. Burhan Turksen, "A greedy variable neighborhood search heuristic for the maximal covering location problem with fuzzy coverage radii," Knowledge-Based Systems, vol. 41, pp. 68-76, 2013.

[7] Y. Hu, Y. Zhang, D. Lamb et al., "Examining and optimizing the BCycle bike-sharing system-a pilot study in Colorado," US. Applied Energy, vol. 247, 2019.

[8] H. Kong, T. Scarlett, and D. Z. S. Jin, "Deciphering the relationship between bikesharing and public transit: modal substitution, integration, and complementation," Transportation Research Part D: Transport and Environment, vol. 85, 2020.

[9] K. Wang and Y.-J. Chen, "Joint analysis of the impacts of built environment on bikeshare station capacity and trip attractions," Journal of Transport Geography, vol. 82, 2020.

[10] Y. Guo and S. Y. He, "Built environment effects on the integration of dockless bike-sharing and the metro," Transportation Research Part D, vol. 83, 2020.

[11] T. Guo, P. Zhang, F. Shao et al., "Allocation optimization of bicycle-sharing stations at scenic spots," Journal of Central South University, vol. 7, 2014. 
[12] M. Benchimol, P. Benchimol, and B. Chappert, "Bike sharing systems: solving the static rebalancing problem," Discrete Optimization, vol. 10, no. 2, pp. 120-146, 2013.

[13] J. P. Romero, A. Ibeas, J. L. Moura, and B. Alonso, "A simulation-optimization approach to design efficient systems of bike-sharing," Procedia-Social and Behavioral Sciences, vol. 54, pp. 646-655, 2012.

[14] I. Benavente and A. Ribeiro, "Bike-sharing stations: a maximal covering location approach," Transportation Research Part A: Policy and Practice, vol. 82, pp. 216-227, 2015.

[15] D. K. George and C. H. Xia, "Fleet-sizing and service availability for a vehicle rental system via closed queueing networks," European Journal of Operational Research, vol. 211, no. 1, pp. 198-207, 2011.

[16] J. C. García-Palomares, J. Gutiérrez, and M. Latorre, "Optimizing the location of stations in bike-sharing programs: a gis approach," Applied Geography, vol. 35, no. 1-2, pp. 235-246, 2012.

[17] L. Liu, L. Sun, Y. Chen et al., "Optimizing fleet size and scheduling of feeder transit services considering the influence of bike-sharing systems," Journal of Cleaner Production, vol. 236, 2019.

[18] C. S. Shui and W. Y. Szeto, "Dynamic green bike repositioning problem - a hybrid rolling horizon artificial bee colony algorithm approach," Transportation Research Part D: Transport and Environment, vol. 60, pp. 119-136, 2018.

[19] A. Kabra, E. Belavina, and K. Girotra, "Bike-share systems: accessibility and availability. working paper," Chicago Booth Research Paper, vol. 15, 2016. 